\title{
Quantum Electrodynamics Based on Self-energy: Lamb Shift and Spontaneous Emission Without Field Quantization
}

\author{
A. O. Barut \\ Jean F. Van Huele \\ vanhuele@byu.edu
}

Follow this and additional works at: https://scholarsarchive.byu.edu/facpub

Part of the Quantum Physics Commons

\section{Original Publication Citation}

Quantum electrodynamics based on self-energy: Lamb shift and spontaneous emission without field quantization. A. O. Barut and J. F. Van Huele. Phys. Rev. A 32, 3187 (1985).

\section{BYU ScholarsArchive Citation}

Barut, A. O. and Van Huele, Jean F., "Quantum Electrodynamics Based on Self-energy: Lamb Shift and Spontaneous Emission Without Field Quantization" (1985). Faculty Publications. 1851.

https://scholarsarchive.byu.edu/facpub/1851 


\title{
Quantum electrodynamics based on self-energy: Lamb shift and spontaneous emission without field quantization
}

\author{
A. O. Barut* \\ Département de Physique Théorique, Université de Genève, 1211 Genève 4, Switzerland \\ J. F. Van Huele ${ }^{\dagger}$ \\ Theoretische Natuurkunde, Vrije Universiteit Brussel, Pleinlaan 2, B-1050 Brussel, Belgium
}

(Received 2 July 1985)

\begin{abstract}
The theory of radiative processes in quantum theory is formulated on the basis of self-energy, in analogy to classical radiation theory, and is explicitly carried out for the calculation of the Lamb shift and spontaneous emission.
\end{abstract}

\section{INTRODUCTION}

In classical electrodynamics the radiative processes are calculated from the self-energy of the electron in external fields. In contrast, in quantum electrodynamics, the selfenergy is first thrown away and one begins with bare particles; then the self-energy is put back in photon by photon, hence the use of perturbation theory. Recently, it has been argued that one ought also to be able to develop a quantum electrodynamics based on the self-energy, ${ }^{1}$ and the general principles of such a theory have been given in the relativistic case. ${ }^{2}$

The purpose of this work is to carry out explicitly the calculations using the full self-energy. We shall derive formulas for the Lamb shift and for spontaneous emission, which are here calculated simultaneously. They are the real and the imaginary parts of a complex energy shift. We use a nonrelativistic wave equation for the electron, as it is simpler to see the main developments. However, it turns out that the final formulas have exactly the same structure for both the nonrelativistic and relativistic cases. In fact the relativistic terms dependent on the spin matrices split nicely. However, we have written this paper so that it can be read independently of the relativistic calculations.

There are several fundamental features of the present theory which one should emphasize at the beginning.

(1) First of all, the matter field $\psi$ is described by a first-quantized wave function. As we shall see, for onebody problems, like the Lamb shift or the anomalous magnetic moment, it is not necessary to quantize the $\psi$ field. The electromagnetic field $A_{\mu}$ is eliminated between the coupled Maxwell-Dirac equations; hence its quantization does not enter into the calculations either. We work throughout with nonperturbative Coulomb-like wave functions, which we never have to expand into "virtual" free-particle states and hence do not need the secondquantization formalism applied to free virtual particles.

(2) The main problem in quantum electrodynamics is to make the renormalization procedure finite. And as expected, the renormalization procedure is quite different for the calculations which starts with the self-energy than for those which use perturbation theory. We separate the self-energy integrals into terms which renormalize the parameters of the theory and other terms which are finite and observables. Actually even the renormalization integrals are finite since they use for example, Coulomb wave functions and not plane waves. The nonlinear equations which include the self-energy can be linearized, after renormalization, by an iterative process that starts from Coulomb wave functions. We find then the formula for the Lamb shift as given by Bethe. ${ }^{3}$ To this lowest order of iteration, which is sufficient for practical purposes, the only infinity comes from the infinitely many Coulomb states of the bare problem. Thus for a system with finitely many states (e.g., a two-level atom) all the results are finite. It can be hoped that even for the Coulomb problem the theory can be made finite if the widths of the states can be taken into account. There is, furthermore, no infrared problem in the present formulation. We have additional terms which after regularization give the vacuum polarization effects.

(3) We proceed from and perform all the calculations in terms of an action rather than the equations of motion. ${ }^{4}$ The theory based on the action is more direct and much simpler, and the bound-state and scattering problems can be treated in a unified manner from the action. In general, the dimensionless action is related to the scattering amplitude $G$ per unit space and time for scattering problems, and to the total invariant energy $\mathscr{E}$ of the system for the bound-state problems as (there is an infinite $\delta$ function proportionality factor)

$$
W_{f i}=(2 \pi)^{4} \delta\left(P_{f}-P_{i}\right) G, \quad W_{f i}=2 \pi \delta\left(E_{f}-E_{i}\right) \mathscr{E} .
$$

(4) Finally, an important feature of the present theory is the simplification it brings to the understanding of radiative processes. All the calculations proceed exactly parallel to classical radiation theory where radiative terms are also obtained nonperturbatively in closed form. ${ }^{5}$ One can attribute quantum effects either to the external quantized field $A^{\text {rad }}$ which has vacuum fluctuations or to the electron itself which has fluctuations due to Zitterbewegung. Here we take the second approach, namely, we assume the electron itself is the source of radiation as well as radia- 
tive corrections. Thus the correct equation of motion for the radiating electron is not the Dirac or Schrödinger equation for a bare electron, but an equation with an additional nonlinear self-energy term, just like the LorentzDirac equation for a classical radiating electron with the Larmor term. There are also a number of significant technical simplifications. One calculates just with wave functions, instead of with field operators.

\section{THE ACTION PRINCIPLE}

For a Schrödinger (or Pauli) field $\phi$ coupled to the electromagnetic field $A_{\mu}$ the action is $\left(c=\hbar=1, d x=d^{4} x\right)$

$$
\begin{aligned}
& W=\int d x\left[i \phi^{*} \partial_{t} \phi-e A^{0} \phi^{*} \phi\right. \\
& \left.-\frac{1}{2 m}(\boldsymbol{\nabla}+i e \mathbf{A}) \phi^{*} \cdot(\boldsymbol{\nabla}-i e \mathbf{A}) \phi-\frac{1}{4} F_{\mu \nu} F^{\mu \nu}\right) \text {. }
\end{aligned}
$$

The Euler-Lagrange equations, when $W$ is varied with respect to $\phi^{*}$ and $A_{\mu}$, are

$$
i \partial_{t} \phi=e A^{0} \phi+\frac{1}{2 m}(-i \boldsymbol{\nabla}-e \mathbf{A}) \cdot(-i \boldsymbol{\nabla}-e \mathbf{A}) \phi
$$

and

$$
F_{, v}^{\mu \nu}=-e j^{\mu}
$$

where the current $j^{\mu}$ has the components

$$
j^{\mu}=\left\{\phi^{*} \phi, \phi * \frac{\overleftrightarrow{\nabla}}{2 m i} \phi-\frac{e}{m} \phi^{*} \phi \mathbf{A}\right\}
$$

By substituting everywhere $(\boldsymbol{\nabla}+i e \mathbf{A}) \cdot \boldsymbol{\sigma}$ for $\boldsymbol{\nabla}+i e \mathbf{A}$ we obtain the Pauli action and the Pauli equations.

In Appendix A we give also a limiting process of how to go from a Dirac action to a Klein-Gordon action and then to the Schrödinger action so that one can carry out the present calculation in any one of these cases.

In what follows, the potential consists of two terms

$$
A_{0}=A_{0}^{E}+A_{0}^{S}, \quad \mathbf{A}=\mathbf{A}^{E}+\mathbf{A}^{S},
$$

an external given field $A^{E}$ which is not a dynamic variable, and a self-field $A^{S}$ originating from the current of the charges which therefore vanishes at infinity. The sources of the external field $A_{\mu}^{E}$ are not included in our problem, hence it can be considered as a solution of the homogeneous Maxwell's equation (excluding the source points). In a genuine two-body problem such as positronium we include also the external field as a dynamic variable.

The contribution of the dynamic field action $-\frac{1}{4} F_{\mu \nu} F^{\mu \nu}$ can be rewritten using Maxwell's equations and the divergence theorem as

$$
\begin{aligned}
-\frac{1}{4} \int d x F_{\mu \nu} F^{\mu \nu}= & -\frac{1}{2} \int d x\left(A_{v}^{S} F^{\mu \nu}\right)_{, \mu}+\frac{1}{2} \int d x A_{v}^{S} F_{, \mu}^{\mu \nu} \\
= & \frac{e}{2} \int d x A_{v}^{S} j^{v} \\
= & \frac{e}{2} \int d x A^{S} \phi^{*} \phi-\frac{e}{2 m i} \int d x \mathbf{A}^{S} \cdot \phi^{*}(\nabla \phi)-\frac{e}{4 m i} \int d x \phi^{*} \phi\left(\nabla \cdot \mathbf{A}^{S}\right) \\
& +\frac{e^{2}}{2 m} \int d x \phi^{*} \phi \mathbf{A}^{E} \cdot \mathbf{A}^{S}+\frac{e^{2}}{2 m} \int d x|\phi|^{2}\left(\mathbf{A}^{S}\right)^{2},
\end{aligned}
$$

where partial integration has been used. Partial integration in the third term of (1) gives, on the other hand,

$$
\int d x \frac{-1}{2 m}(\boldsymbol{\nabla}+i e \mathbf{A}) \phi^{*} \cdot(\boldsymbol{\nabla}-i e \mathbf{A}) \phi=\int d x \frac{1}{2 m} \phi^{*}\left(\Delta-i e \boldsymbol{\nabla} \cdot \mathbf{A}-2 i e \mathbf{A} \cdot \boldsymbol{\nabla}-e^{2} A^{2}\right) \phi
$$

From (6) and (7) the total action can be written in terms of $\phi$ and $A_{\mu}$ 's only. The final result is

$$
\begin{aligned}
W=\int d x \phi^{*}[ & i \partial_{t} \phi-e A_{0}^{E} \phi-\frac{e}{2} A_{0}^{S} \phi+\frac{\Delta}{2 m} \phi-\frac{i e}{2 m}\left(\nabla \cdot \mathbf{A}^{E}\right) \phi-\frac{i e}{4 m}\left(\nabla \cdot \mathbf{A}^{S}\right) \phi-\frac{i e}{m} \mathbf{A}^{E} \cdot(\nabla \phi)-\frac{i e}{2 m} \mathbf{A}^{S \cdot(\nabla \phi)} \\
& \left.-\frac{e^{2}}{2 m}\left(A^{E}\right)^{2} \phi-\frac{e^{2}}{2 m} \mathbf{A}^{E} \cdot \mathbf{A}^{S} \phi\right]
\end{aligned}
$$


Had we started from the Pauli action the commutation relations for the operators $\mathbf{p}, \boldsymbol{\sigma}, \mathbf{A}$ would have led to the additional term

$$
\begin{aligned}
& W^{\text {Pauli }}-W^{\text {Schröd }}=\int d x \phi^{*}\left(-\frac{e}{2 m} \sigma \cdot\left(\nabla \times \mathbf{A}^{E}\right)\right. \\
& -\frac{e}{4 m} \boldsymbol{\sigma} \cdot\left(\boldsymbol{\nabla} \times \mathbf{A}^{S}\right) \boldsymbol{\phi} .
\end{aligned}
$$

\section{ELIMINATION OF THE SELF-FIELD}

The first step in our study of the action $W$ in (8) is to eliminate the self-field $A_{\mu}^{s}$, i.e., to express it in terms of the current $j_{\mu}$ of the $\phi$ field by the formulas

$$
A_{\mu}^{S}(x)=e \int d x^{\prime} D_{\mu v}\left(x-x^{\prime}\right) j^{v}\left(x^{\prime}\right)
$$

This formula is derived and discussed in Appendix B, where we also give the explicit form of the Green's functions $D_{\mu v}\left(x-x^{\prime}\right)$ in different gauges. The final result is, as it should be, gauge invariant. We shall choose the Coulomb gauge (B12) in order to compare our results with the standard nonrelativistic calculations of the Lamb shift.

By inserting (10) into (8) we have expressed the action $W$ (besides the given external field $A_{\mu}^{E}$ ) entirely in terms of the matter field $\phi$, which now has nonlinear and nonlocal terms coming from Eq. (10). We shall write these terms in the next section in terms of a set of Fourier coefficients.

\section{FOURIER EXPANSION OF THE TOTAL MATTER FIELD}

The action $W$, being an integral over all space and time, expresses all possible interactions between the matter field $\phi$ and electromagnetic field $A_{\mu}$. It guarantees that the equations of motion are consistent with the conservation laws. In order to separate individual processes of definite energies we perform, as a second step in our analysis, a Fourier expansion of $\phi$ in the time coordinate:

$$
\phi(\mathbf{x}, t)=\mathfrak{f}_{n} \phi_{n}(\mathbf{x}) e^{-i E_{n} t} .
$$

We shall show that the coefficients $\phi_{n}$ correspond to the physical wave functions. The action expressed in terms of the Fourier components $\phi_{n}$, yet to be determined, becomes, using (B12),

$$
\begin{aligned}
W= & \int d x f_{n, m} \phi_{n}^{*}(\mathbf{x}) e^{i E_{n} t} X_{1} \phi_{m}(\mathbf{x}) e^{-i E_{m} t} \\
& -\frac{1}{(2 \pi)^{4}} \int d x d x^{\prime} d k f_{n, m, r, s} \phi_{n}^{*}(\mathbf{x}) e^{i E_{n} t} e^{i E_{r} t^{\prime}} X_{2} e^{-i E_{m} t} e^{-i E_{s} t^{\prime}} e^{-i k\left(x-x^{\prime}\right)} \phi_{m}(\mathbf{x})+\cdots
\end{aligned}
$$

where

$$
\begin{aligned}
X_{1}= & E_{m}-e A_{0}^{E}+\frac{\Delta}{2 m}-\frac{i e}{2 m}\left(\nabla \cdot \mathbf{A}^{E}\right) \\
& -\frac{i e}{m} \mathbf{A}^{E} \cdot(\nabla)-\frac{e^{2}}{2 m}\left(\mathbf{A}^{E}\right)^{2}
\end{aligned}
$$

and

$$
\begin{aligned}
X_{2}= & -\frac{e^{2}}{2} \frac{1}{\mathbf{k}^{2}} \phi_{r}^{*}\left(\mathbf{x}^{\prime}\right) \phi_{s}\left(\mathbf{x}^{\prime}\right) \\
& +\left[\phi_{r}^{*}\left(\mathbf{x}^{\prime}\right) \frac{\overleftrightarrow{\nabla}}{2 m i} \phi_{s}\left(\mathbf{x}^{\prime}\right)-\frac{e}{m} A_{l}^{E} \phi_{r}^{*}\left(\mathbf{x}^{\prime}\right) \phi_{s}\left(\mathbf{x}^{\prime}\right)\right] \\
& \times\left[-\frac{i e^{2}}{2 m} \frac{\nabla_{j}}{k^{2}}-\frac{e^{3}}{2 m} \frac{A_{j}^{E}}{k^{2}}\right]\left[\delta_{j l}-\frac{k_{j} k_{l}}{\mathbf{k}^{2}}\right] .
\end{aligned}
$$

Contrary to the relativistic case, the nonrelativistic current (5) still contains the field $A_{\mu}$. Strictly speaking (B2) is still an integral equation to be solved for $A_{\mu}$. In this respect the relativistic problem is much simpler. However, the terms in $W$ coming from this $A$ dependence of the current are successively higher order in $e^{2}$; hence the action can be truncated in an iterative solution. In the case of an external field which has only a zero component $A^{0 E}$, like the Coulomb potential of an $\mathrm{H}$ atom, this complication does not arise. In what follows we shall set $\mathbf{A}^{E}=0$ and obtain

$$
\begin{aligned}
X_{1}= & E_{m}-e A_{0}^{E}+\frac{\Delta}{2 m}, \\
X_{2}= & -\frac{e^{2}}{2} \frac{1}{\mathbf{k}^{2}} \phi_{r}^{*}\left(\mathbf{x}^{\prime}\right) \phi_{s}\left(\mathbf{x}^{\prime}\right) \\
& -\frac{i e^{2}}{2 m} \frac{1}{k^{2}}\left[\delta_{j l}-\frac{k_{j} k_{l}}{\mathbf{k}^{2}}\right]\left[\phi_{r}^{*}\left(\mathbf{x}^{\prime}\right) \frac{\overleftrightarrow{\nabla}_{l}}{2 m i} \phi_{s}\left(\mathbf{x}^{\prime}\right)\right] \nabla_{j}, \\
X_{3}= & X_{4}=\cdots=0 .
\end{aligned}
$$




\section{TIME INTEGRATIONS}

In the third step of our analysis we perform the integrations over $d x^{0}, d x^{0^{\prime}}$, and $d k^{0}$ on $W$ and obtain

$$
\begin{aligned}
W^{(1)} & =2 \pi \int d \mathbf{x} \boldsymbol{\xi}_{n, m} \phi_{n}^{*}(\mathbf{x}) X_{1} \phi_{m}(\mathbf{x}) \delta\left(E_{n}-E_{m}\right) \\
& =\sum_{n, m} W_{n m}^{(1)}=\sum_{n, m} 2 \pi \delta\left(E_{n}-E_{m}\right) \mathscr{E}_{n m}^{(1)}
\end{aligned}
$$

or, using the relation between the action $W$ and energy $\mathscr{E}$,

$$
\mathscr{E}^{(1)}=\int d \mathbf{x} \mathcal{F}_{n} \phi_{n}^{*}(\mathbf{x}) X_{1} \phi_{n}(\mathbf{x})=\sum_{n, m} \mathscr{C}_{n n}^{(1)} \delta_{n m}
$$

and

$$
\begin{aligned}
W^{(2)} & =-\frac{(2 \pi)^{2}}{(2 \pi)^{4}} \int d \mathbf{x} d \mathbf{x}^{\prime} d \mathbf{k} \oint_{n, m, r, s} d k^{0} \delta\left(E_{n}-E_{m}-k^{0}\right) \delta\left(E_{r}-E_{s}+k^{0}\right) e^{i \mathbf{k} \cdot\left(\mathbf{x}-\mathbf{x}^{\prime}\right)} \phi_{n}^{*}(\mathbf{x}) X_{2} \phi_{m}(\mathbf{x}) \\
& =-\frac{1}{(2 \pi)^{2}} \int d \mathbf{x} d \mathbf{x}^{\prime} d \mathbf{k} \oint_{n, m, r, s} \delta\left(E_{n}-E_{m}+E_{r}-E_{s}\right) e^{i \mathbf{k} \cdot\left(\mathbf{x}-\mathbf{x}^{\prime}\right)} \phi_{n}^{*}(\mathbf{x}) X_{2}\left(k^{0}=E_{n}-E_{m}\right) \phi_{m}(\mathbf{x}) .
\end{aligned}
$$

Since the $d t$ and $d t^{\prime}$ integrations are done before the $k^{0}$ integration, the usual problem of choice of a contour for the $k^{0}$ integration does not arise.

From the appearance of the $\delta$ functions of energy in (14) we see that the first term $W^{(1)}$, with (13), is a single sum of actions of the electron in a Coulomb field over the energies $E_{m}$. Note, however, that $\phi_{m}$ and $E_{m}$ do not yet have the precise Coulomb values, for there is a second term, and the whole action is still exact. The variation of the action $W$ with respect to the $\phi_{n}$ 's would lead to an infinite set of coupled nonlinear equations.

In the second term $W^{(2)}$ the factor $\delta\left(E_{n}-E_{m}+E_{r}-E_{s}\right)$ tells us that the summations must be restricted to those values for which $E_{n}-E_{m}+E_{r}-E_{s}$ $=0$. We wish to bring the second term $W^{(2)}$ to the form of $W^{(1)}$ with a factor $\delta\left(E_{n}-E_{m}\right)$ so that we can isolate the corrections to the energies of a level $m$. This can be done in two ways (as was already introduced in relativistic case $^{2}$ ):

$$
\begin{aligned}
& E_{n}=E_{s}, \text { hence } E_{m}=E_{r} \\
& E_{n}=E_{m}, \text { hence } E_{s}=E_{r} .
\end{aligned}
$$

There is a term $E_{n}=E_{s}=E_{m}=E_{r}$ common to both of these cases. (For a discussion of other solutions of $E_{n}-E_{m}+E_{r}-E_{s}=0$ see Appendix C.) We separate terms in $W^{(2)}$ satisfying (15a) and (15b), change dummy indices, and eliminate the $\delta$ function $\delta(0)$ by passing from the dimensionless $W$ to the total energy of the system:

$$
\begin{aligned}
& 2 \pi \mathscr{E}^{(2)}=\frac{e^{2}}{2(2 \pi)^{2}} \mathcal{E}_{n, m} d \mathbf{k} \frac{1}{\mathbf{k}^{2}}{ }_{n} T_{m}{ }^{0}(\mathbf{k})_{m} T_{n}{ }^{0}(-\mathbf{k})+\frac{e^{2}}{2(2 \pi)^{2}} \mathcal{f}_{(\substack{n \neq m \\
n \neq m}} \frac{d \mathbf{k}}{\mathbf{k}^{2}}{ }_{n} T_{n}{ }^{0}(\mathbf{k})_{m} T_{m}(-\mathbf{k}) \\
& -\frac{2 e^{2}}{(2 m)^{2}(2 \pi)^{2}} \xi_{n, m} d \mathbf{k} \frac{{ }_{n} T_{m}^{j}(\mathbf{k})_{m} T_{n}{ }^{l}(-\mathbf{k})}{\left(E_{n}-E_{m}\right)^{2}-\mathbf{k}^{2}}\left(\delta_{j l}-\frac{k_{j} k_{l}}{\mathbf{k}^{2}}\right) \\
& -\frac{2 e^{2}}{(2 m)^{2}(2 \pi)^{2}} \boldsymbol{\xi}_{n, m} d \mathbf{k} \frac{{ }_{n} T_{n}{ }^{j}(\mathbf{k})_{m} T_{m}{ }^{l}(-\mathbf{k})}{-\mathbf{k}^{2}}\left[\delta_{j l}-\frac{k_{j} k_{l}}{\mathbf{k}^{2}}\right),
\end{aligned}
$$

where we have introduced the form factors

$$
\begin{aligned}
& { }_{n} \mathbf{T}_{m}(\mathbf{k}) \equiv \int d \mathbf{x} \phi_{n}^{*}(\mathbf{x}) \frac{\nabla}{i} \phi_{m}(\mathbf{x}) e^{i \mathbf{k} \cdot \mathbf{x}}, \\
& { }_{n} T_{m}{ }^{0}(\mathbf{k}) \equiv \int d \mathbf{x} \phi_{n}^{*}(\mathbf{x}) \phi_{m}(\mathbf{x}) e^{i \mathbf{k} \cdot \mathbf{x}}
\end{aligned}
$$

The last term in $W^{(2)}$ vanishes, because ${ }_{n} T_{n}{ }^{i}(\mathbf{k})=0$.
Indeed

$$
{ }_{n} T_{n}{ }^{i}(\mathbf{k}) \sim \phi_{n}^{*} \frac{\nabla^{i}}{2} \phi_{n} \sim \operatorname{Im}\left(\phi_{n}^{*} \nabla^{i} \phi_{n}\right)
$$

and vanishes for even magnetic quantum numbers $\left(\phi_{n}\right.$ real) and for odd magnetic quantum numbers the two terms with opposite magnetic quantum numbers cancel.

Finally, as shown in Appendix D, the angular integrations $\int d \Omega_{k}$ give a factor of $2 \pi, 2 \pi$, and $8 \pi / 3$, respec- 
tively, in the three remaining terms in $\mathscr{E}^{(2)}$ and we obtain

$$
\begin{aligned}
2 \pi \mathscr{C}^{(2)}= & \frac{e^{2}}{4 \pi} \xi_{n, m} \int d k_{n} T_{m}{ }^{0}(\mathbf{k})_{m} T_{n}{ }^{0}(-\mathbf{k}) \\
& +\frac{e^{2}}{4 \pi} \oint_{n, m} \int d k_{n} T_{n}{ }^{0}(\mathbf{k})_{m} T_{m}{ }^{0}(-\mathbf{k}) \\
& -\frac{2}{3} \frac{2 e^{2}}{4 \pi} \frac{1}{m^{2}} \oint_{n, m} \int d k k^{2} \frac{{ }_{n} \mathbf{T}_{m}(\mathbf{k}){ }_{m} \mathbf{T}_{n}(-\mathbf{k})}{\left(E_{n}-E_{m}\right)^{2}-k^{2}}
\end{aligned}
$$

\section{SEPARATION OF RENORMALIZATION AND OBSERVABLE TERMS}

Recall that in classical electrodynamics the self-energy term in the equation of motion can be split into two parts: The first part has the form of an inertial mass term which will be added to the bare mass term $m_{0} \ddot{z}_{\mu}$ to get the re- normalized mass term $\left(m_{0}+\delta m\right) \ddot{z}_{\mu}=m \ddot{z}_{\mu}$, and the second term is finite and observable. We shall proceed similarly here to identify parts of $\mathscr{E}^{(2)}$ which renormalize $\mathscr{C}^{(1)}$ and the remaining parts which are finite and observable.

First we split the denominator in the last term of $\mathscr{E}^{(2)}$ by the well-known identity

$$
\begin{aligned}
\frac{1}{\left(E_{n}-E_{m}\right)^{2}-k^{2}}= & \frac{1}{2 k}\left[\frac{1}{E_{n}-E_{m}-k}-\frac{1}{E_{n}-E_{m}+k}\right] \\
=\frac{1}{2 k^{2}} & {\left[\frac{E_{n}-E_{m}}{E_{n}-E_{m}-k}\right.} \\
& \left.+\frac{E_{m}-E_{n}}{E_{m}-E_{n}-k}-2\right]
\end{aligned}
$$

and obtain for the total energy the five terms $\left(\alpha=e^{2} 4 \pi\right)$

$$
\begin{aligned}
& \mathscr{E}=\mathscr{C}_{1}+\mathscr{C}_{2}+\mathscr{C}_{3}+\mathscr{C}_{4}+\mathscr{C}_{5} \\
& =\xi_{n} \int d \mathbf{x} \phi_{n}^{*}(\mathbf{x})\left[E_{n}-e A_{0}^{E}+\frac{\Delta}{2 m}\right] \phi_{n}(\mathbf{x})+\xi_{n, m} \frac{\alpha}{2 \pi} \int d k_{n} T_{m}{ }^{0}(\mathbf{k})_{m} T_{n}{ }^{0}(-\mathbf{k}) \\
& +\xi_{n, m} \frac{\alpha}{2 \pi} \int d k_{n} T_{n}{ }^{0}(\mathbf{k})_{m} T_{m}{ }^{0}(-\mathbf{k})+\xi_{n, m}\left[-\frac{2}{3} \frac{\alpha}{\pi} \frac{1}{m^{2}} \int d k \frac{E_{n}-E_{m}}{E_{n}-E_{m}-k}{ }_{n} \mathbf{T}_{m}(\mathbf{k}) \cdot{ }_{m} \mathbf{T}_{n}(-\mathbf{k})\right] \\
& +\xi_{n, m} \frac{2}{3} \frac{\alpha}{\pi} \frac{1}{m^{2}} \int d k_{n} \mathbf{T}_{m}(\mathbf{k}) \cdot_{m} \mathbf{T}_{n}(-\mathbf{k}) .
\end{aligned}
$$

In the last term of (18), since we have a double sum $f_{n, m}$ and since ${ }_{n} \mathbf{T}_{m}(\mathbf{k}){ }_{m} \mathbf{T}_{n}(-\mathbf{k})$ is invariant under the permutation $n \leftrightarrow m$, we have used the identity.

$$
\begin{aligned}
\sum_{n, m} \frac{1}{2 k^{2}}\left[\frac{E_{n}-E_{m}}{E_{n}-E_{m}-k}\right. & \left.+\frac{E_{m}-E_{n}}{E_{m}-E_{n}-k}-2\right) \\
= & \sum_{n, m} \frac{1}{k^{2}}\left[\frac{E_{n}-E_{m}}{E_{n}-E_{m}-k}-1\right] .
\end{aligned}
$$

Our energy $\mathscr{C}$ in (20) expresses the energies and energy shifts of all levels, that is, the total calorimetric energy shift of the system. The first term is a single sum; all other terms involve a double sum and contain the shift of a level $n$ due to a level $m$, as well as a shift of the level $m$ due to $n$. In order to isolate a particular level shift we write the total energy in the form

$$
\mathscr{C}=\sum_{n} \mathscr{E}^{(n)}
$$

Now the first term in (20) is already in this form. In the second term we assume the completeness relations which we justify a posteriori

$$
\begin{aligned}
\mathscr{C}_{2}^{(n)}= & \frac{\alpha}{2 \pi} \int d \mathbf{x} d \mathbf{x}^{\prime} d k \sum_{m} \phi_{n}\left(\mathbf{x}^{\prime}\right) \phi_{m}\left(\mathbf{x}^{\prime}\right) e^{i \mathbf{k} \cdot \mathbf{x}^{\prime}} \\
& \times \phi_{m}(\mathbf{x}) \phi_{n}(\mathbf{x}) e^{-i \mathbf{k} \cdot \mathbf{x}} \\
= & \frac{\alpha}{2 \pi} \int d \mathbf{x} d \mathbf{x}^{\prime} d k \phi_{n}^{*}\left(\mathbf{x}^{\prime}\right) \delta\left(\mathbf{x}-\mathbf{x}^{\prime}\right) \phi_{n}(x) e^{i \mathbf{k} \cdot\left(\mathbf{x}-\mathbf{x}^{\prime}\right)} \\
= & \frac{\alpha}{2 \pi} \int d k d \mathbf{x} \phi_{n}^{*}(\mathbf{x}) \phi_{n}(\mathbf{x})=\int d \mathbf{x} \phi_{n}^{*}(\mathbf{x}) \Lambda \phi_{n}(\mathbf{x}),
\end{aligned}
$$

$\frac{\alpha}{2 \pi} \int_{0}^{\infty} d k=\Lambda$

Thus (23) is a constant infinite term, the same for all levels $n$ (which will of course drop out in the measured energy differences between two levels). The third term is the sum of static Coulomb self-energies of levels. Indeed 


$$
\begin{aligned}
\mathscr{C}_{3}^{(n)}= & \frac{\alpha}{2 \pi} \int d \mathbf{x} d \mathbf{x}^{\prime} d k{\underset{(}{(m \neq n)}}_{\substack{m, n \\
m_{n}}} \phi_{n}^{*}(\mathbf{x}) \phi_{n}(\mathbf{x}) e^{-i \mathbf{k} \cdot \mathbf{x}} \\
& \times \phi_{m}^{*}\left(x^{\prime}\right) \phi_{m}\left(x^{\prime}\right) e^{i \mathbf{k} \cdot \mathbf{x}^{\prime}} \\
= & \frac{\alpha}{2 \pi} \int d \mathbf{x} d \mathbf{x}^{\prime} \sum_{m} \phi_{n}^{*}(\mathbf{x}) \phi_{n}(\mathbf{x}) \frac{1}{\left|\mathbf{x}-\mathbf{x}^{\prime}\right|} \phi_{m}^{*}\left(\mathbf{x}^{\prime}\right) \phi_{m}\left(\mathbf{x}^{\prime}\right) \\
= & \frac{\alpha}{2 \pi} \int d \mathbf{x}^{\prime} d \mathbf{x} \sum_{m} \frac{\rho_{n}(\mathbf{x}) \rho_{m}\left(\mathbf{x}^{\prime}\right)}{\left|\mathbf{x}-\mathbf{x}^{\prime}\right|} \\
= & \int d \mathbf{x} \phi_{n}^{*}(\mathbf{x})\left[\frac{\alpha}{2 \pi} \sum_{m} \int d \mathbf{x}^{\prime} \frac{\rho_{m}\left(\mathbf{x}^{\prime}\right)}{\left|\mathbf{x}-\mathbf{x}^{\prime}\right|}\right] \phi_{n}(\mathbf{x})
\end{aligned}
$$

The relativistic counterpart of this term ${ }^{2}$ is precisely the vacuum polarization term studied by Wichmann and
Kroll, ${ }^{6}$ who extract after regularization the finite Uehling potential $^{7}$ which causes a small level shift to be added to the Bethe term. Wichmann and Kroll interpret the negative energy states as the scattering states of the positrons from the proton. In the present nonrelativistic calculation we could also introduce positron scattering states in the Coulomb field. Work is in progress to regularize the integral (24) in the nonrelativistic case. We denote the regularized finite part of (24) as

$$
\mathscr{C}_{3}^{(n) \mathrm{reg}}=\int d \mathbf{x} \phi_{n}^{*}(\mathbf{x}) U(x) \phi_{n}(\mathbf{x}) .
$$

Finally the fourth term represents both the Bethe term as well as spontaneous emission. Using

$$
\frac{1}{E_{n}-E_{m}-k}=P \frac{1}{E_{n}-E_{m}-k}-i \pi \delta\left(E_{n}-E_{m}-k\right)
$$

these two terms are

$$
\begin{aligned}
\mathscr{E}_{4}^{(n)}= & -\frac{2}{3} \frac{\alpha}{\pi} \frac{1}{m^{2}} \oint_{m}\left(E_{n}-E_{m}\right) \int d k \frac{1}{E_{n}-E_{m}-k}{ }_{n} \mathbf{T}_{m}(\mathbf{k}){ }_{m} \mathbf{T}_{n}(-\mathbf{k}) \\
& +i \frac{2}{3} \frac{\alpha}{\pi} \frac{\pi}{m^{2}} \oint_{m(<n)}\left(E_{n}-E_{m}\right) \int d k \delta\left(E_{n}-E_{m}-k\right)_{n} \mathbf{T}_{m}(\mathbf{k}){ }_{m} \mathbf{T}_{n}(-\mathbf{k})
\end{aligned}
$$

In the first term we sum over all discrete and continuous states, in the second over $m$ such that $E_{m}<E_{n}$. Hence the ground state is stable. Since $k$ is integrated over nonnegative values the imaginary part $i \pi \delta\left(E_{n}-E_{m}-k\right)$ in (26) will contribute only if $E_{n}-E_{m}>0$. If we choose a negative imaginary part in (26), the second term in (27) describes the decay of a higher state; if we choose the positive imaginary part, it will be interpreted as the swelling of a lower state in the total action.

Note that in the dipole approximation (DA) Eq. (27) becomes the familiar formula

$$
\begin{aligned}
\mathscr{C}_{4}^{(n) \mathrm{DA}}= & -\frac{2}{3} \frac{\alpha}{\pi} \frac{1}{m^{2}} \boldsymbol{f}_{m}\left(E_{n}-E_{m}\right) \int d k \frac{\mathbf{p}_{n m}^{*} \cdot \mathbf{p}_{m n}}{E_{n}-E_{m}-k} \\
& +\frac{2}{3} i \frac{\alpha}{m^{2}} \oint_{m(<n)}\left(E_{n}-E_{m}\right) \mathbf{p}_{n m}^{*} \cdot \mathbf{p}_{m n} .
\end{aligned}
$$

Using the relations

$$
\mathbf{p}_{n m}=i\left(E_{n}-E_{m}\right) m \mathbf{r}_{n m}
$$

between the matrix elements of $\mathbf{r}$ and $\mathbf{p}$, the last term becomes

$$
\operatorname{Im} \mathscr{E}_{4}^{(n)}=\frac{2 \alpha}{3} \sum_{m}\left(E_{n}-E_{m}\right)^{3}\left|r_{n m}\right|^{2} .
$$

Since an excited state decays like $\exp [-i(E-i \Gamma / 2) t]{ }^{8}$ we get for the decay rate $\Gamma=2 \operatorname{Im} \mathscr{C}_{4}$, which is the same as the Einstein $A$ coefficient $^{9}$ (or inverse mean lifetime $\Gamma=A=1 / \tau)$.

It is known, however, that the dipole formula (28) leads to additional infinites. ${ }^{3}$ It is better to use the exact equation (27). For scattering states Eq. (27) also gives the probability of bremsstrahlung in Coulomb or external fields.

Finally, the fifth and the last term in the action (23) gives, assuming again the completeness relation which we will justify a posteriori,

$$
\begin{aligned}
\mathscr{E}_{5}^{(n)} & =\frac{2}{3} \frac{\alpha}{\pi m^{2}} \oint_{m} \int d k d \mathbf{x} d \mathbf{x}^{\prime} e^{i \mathbf{k} \cdot \mathbf{x}^{\prime}} \phi_{n}^{*}\left(\mathbf{x}^{\prime}\right) \frac{\nabla}{i} \phi_{m}\left(\mathbf{x}^{\prime}\right) \phi_{m}^{*}(\mathbf{x}) \cdot \frac{\nabla}{i} \phi_{n}(\mathbf{x}) e^{-i \mathbf{k} \cdot \mathbf{x}} \\
& =\frac{2 \alpha}{3 \pi m^{2}} \int d k d \mathbf{x} d \mathbf{x} e^{-i \mathbf{k} \cdot\left(\mathbf{x}-\mathbf{x}^{\prime}\right)} \phi_{n}^{*}\left(\mathbf{x}^{\prime}\right) \frac{\nabla}{i} \delta\left(\mathbf{x}-\mathbf{x}^{\prime}\right) \cdot \frac{\nabla}{i} \phi_{n}(\mathbf{x}) \\
& =-\frac{2 \alpha}{3 \pi m^{2}} \int d k d \mathbf{x} \phi_{n}^{*}(\mathbf{x}) \Delta \phi_{n}(\mathbf{x}) \\
& =-\frac{2 \alpha}{3 \pi m^{2}} \Lambda \int d \mathbf{x} \phi_{n}^{*}(\mathbf{x}) \Delta \phi_{n}(\mathbf{x})
\end{aligned}
$$


This is a contribution to the kinetic energy, and hence to the mass renormalization.

Collecting terms, the total energy $\mathscr{C}$ now has the form

$$
\begin{aligned}
\mathscr{E}=\mathcal{F}_{n} d \mathbf{x} \phi_{n}^{*}(\mathbf{x})[ & E_{n}+\frac{\alpha}{2 \pi} \Lambda+\frac{\Delta}{2 m} \\
& \left.-\Delta \frac{2 \alpha \Lambda}{3 \pi m^{2}}-\frac{Z e^{2}}{r}+U\right] \phi_{n}(\mathbf{x}) \\
& \left.-\int d \mathbf{x}^{\prime} L\left(\mathbf{x}, \mathbf{x}^{\prime}\right) \phi_{n}\left(\mathbf{x}^{\prime}\right)\right]
\end{aligned}
$$

where $L$ is the operator defined by (27), or

$$
\mathscr{E}=\mathcal{E}_{n} d \mathbf{x} \phi_{n}^{*}(\mathbf{x})\left[\widetilde{E}_{n}+\frac{\Delta}{2 \widetilde{m}}+V-\widetilde{L}\right] \phi_{n}(\mathbf{x})
$$

with the definitions

$\widetilde{E}_{n}=E_{n}+\frac{\alpha}{2 \pi} \Lambda, \frac{1}{2 \tilde{m}}=\frac{1}{2 m}-\frac{2 \alpha \Lambda}{3 \pi m^{2}}, \quad V=-\frac{Z e}{r}+U$,

$\int d \mathbf{x}^{\prime} L\left(\mathbf{x}, \mathbf{x}^{\prime}\right) \phi_{n}\left(\mathbf{x}^{\prime}\right)=\widetilde{L}(x) \phi_{n}(\mathbf{x})$.

The Lamb-shift operator $\widetilde{L}$ is nonlocal, as it should be from the character of the self-action.

We have not yet said anything about the functions $\phi_{n}$. The only property we used was their completeness, so that Eq. (32) is still exact, but $\widetilde{L}$ contains all the $\phi_{m}$ 's in a nonlinear way as given in (27). Anticipating that the expectation value of $\widetilde{L}$ is small after renormalization we see from (32) that we can now choose $\phi_{n}$ to be the solutions of the renormalized Coulomb problem (hence they are complete). If we write

$$
\widetilde{E}_{n}=E_{n}^{\text {Coul }}+\Delta E
$$

then

$$
\left[E_{n}^{\text {Coul }}+\frac{\Delta}{2 \tilde{m}}-\frac{Z e^{2}}{r}\right] \phi_{n}^{\text {Coul }}=0 .
$$

Since the total action is zero when the equations of motion are substituted (extremum of $W$ ), the vanishing of the square brackets in (32) gives

$$
\left\langle\Delta E_{n}\right\rangle=\langle n|(\tilde{L}-U)| n\rangle .
$$

Consequently we can go back and perform the integrals (20), including renormalization integrals for $\Lambda$ and $U$, with physical Coulomb wave functions. The changes in the wave function $\phi_{n}$ are, as usual, corrections of higher order than the energy shift. This completes the calculations of the Lamb shift and spontaneous emission.

\section{CONCLUDING REMARKS}

We have presented an alternate point of view to the treatment of radiative processes in quantum electrodynamics. New formulations can be useful even for phenomena which have been studied as long as the Lamb shift. In fact, the question has been raised many times whether one can calculate the Lamb shift without field quantization. Our program is to see how far we can go in understanding radiative processes from the point of view of self-energy rather than from the point of view of an assumed quantized radiation field. This is a legitimate point of view and the one used in classical electrodynamics.

The salient features of our theory, besides its conceptual simplicity-in our opinion-are the following.

(i) A joint treatment of the energy shift and the spontaneous emission and the derivation of the corresponding effective potentials [and clearly the inclusion of the anomalous magnetic moment if the $T$ 's in Eq. (27) are chosen to be the relativistic form factors].

(2) The new renormalization procedure in Eqs. (32) and (33) showing why and how we choose $\phi_{n}$ as the renormalized Coulomb solutions, and the introduction of the nonlocal $L$ operator.

(3) Explanation of how the decaying of an excited state and the growing of lower states are contained in the total action, and a clear interpretation of the passage from the quadratic propagator $1 /\left[\left(E_{n}-E_{m}\right)^{2}-k^{2}\right]$ to the linear form $1 /\left(E_{n}-E_{m}-k\right)$.

(4) A unified description of relativistic and nonrelativistic regimes; only the form of the form factors $T$ changes, otherwise all the formulas hold for any form of the current in the theory. In the standard treatment a lowenergy calculation with a cutoff is matched with an entirely different high-energy calculation. Further, each individual contribution to the energy shift is finite, the only remaining infinity comes from the infinite number of Coulomb states.

\section{ACKNOWLEDGMENT}

One of us (A.O.B.) would like to acknowledge partial support from the Swiss National Science Foundation.

\section{APPENDIX A}

We show here the appropriate substitutions and limits used to go from the Dirac to the Klein-Gordon action and then further to the Schrödinger action. These are valid also for field equations and currents.

Dirac to Klein-Gordon:

$$
\begin{aligned}
& \psi_{\mathrm{D}} \rightarrow \sqrt{m} \phi_{\mathrm{KG}}, \bar{\psi}_{\mathrm{D}} \rightarrow \sqrt{m} \phi_{\mathrm{KG}}^{*}, \\
& \gamma^{\mu} \rightarrow \frac{i}{2 m} \overleftrightarrow{\partial}^{\mu}-\frac{e}{m} A^{\mu}, \\
& \phi^{*} \overleftrightarrow{\partial}^{\mu} \phi \equiv \phi^{*}\left(\partial^{\mu} \phi\right)-\left(\partial^{\mu} \phi^{*}\right) \phi, D^{\mu} \equiv \partial^{\mu}+i e A^{\mu}, \\
& \bar{\psi}_{\mathrm{D}}\left[i \gamma^{\mu}\left[\frac{\overleftrightarrow{\partial}_{\mu}}{2}+i e A_{\mu}\right]-m\right] \psi_{\mathrm{D}} \\
& \rightarrow \phi_{\mathrm{KG}}^{*}\left(-\overleftrightarrow{\mathrm{D}}^{\mu} \overleftrightarrow{\mathrm{D}}_{\mu}-m^{2}\right) \phi_{\mathrm{KG}}
\end{aligned}
$$

Klein-Gordon to Schrödinger:

$$
\begin{aligned}
& \phi_{\mathrm{KG}} \rightarrow \sqrt{2 m} e^{-i m c t} \phi_{\mathrm{S}} \\
& \phi_{\mathrm{KG}}^{*} \rightarrow \sqrt{2 m} e^{i m c t} \phi_{\mathrm{S}}^{*}, \\
& c \rightarrow \infty,
\end{aligned}
$$




$$
\begin{aligned}
\phi_{\mathrm{KG}}^{*}( & \left.-\overleftrightarrow{\mathrm{D}}^{\mu} \overleftrightarrow{\mathrm{D}}_{\mu}-m^{2}\right) \phi_{\mathrm{KG}} \\
\rightarrow & \phi_{\mathrm{S}}^{*}\left(\frac{i}{c} \partial_{t}-\frac{e}{c} A^{0}-\frac{1}{2 m}(\nabla-i e \mathbf{A})^{2}\right) \phi_{\mathrm{S}}
\end{aligned}
$$

\section{APPENDIX B}

The self-potential of the electron satisfies Maxwell's equations:

$$
\partial^{v} \partial_{v} A_{\mu}^{S}(x)-\partial^{v} \partial_{\mu} A \underset{v}{S}(x)=e j_{\mu}(x)
$$

which we can solve formally,

$$
A_{\mu}^{S}(x)=e \int d x^{\prime} D_{\mu v}\left(x-x^{\prime}\right) j^{v}\left(x^{\prime}\right)+A_{\mu}^{\text {in }}(x)
$$

provided that

$$
\int d x^{\prime}\left[\partial^{v} \partial_{v} D_{\mu \rho}\left(x-x^{\prime}\right)-\partial^{v} \partial_{\mu} D_{v \rho}\left(x-x^{\prime}\right)\right] j^{\rho}\left(x^{\prime}\right)=j_{\mu}(x)
$$

or

$\partial^{v} \rho_{v} D_{\mu \rho}\left(x-x^{\prime}\right)-\partial^{v} \partial_{\mu} D_{v \rho}\left(x-x^{\prime}\right)=g_{\mu \rho} \delta^{4}\left(x-x^{\prime}\right)$.

This equation can be solved once a particular gauge is chosen. We give the expression for $D_{\mu v}\left(x-x^{\prime}\right)$ in three different gauges.

\section{The Lorentz gauge}

The condition on the potential $\partial^{\mu} A_{\mu}^{S}(x)=0$ implies

$$
\int d x^{\prime} \partial^{\mu} D_{\mu v}\left(x-x^{\prime}\right) j^{v}\left(x^{\prime}\right)=0
$$

and transforms (B1) into

$$
\int d x^{\prime} \partial^{\rho} \partial_{\rho} D_{\mu \nu}\left(x-x^{\prime}\right) j^{v}\left(x^{\prime}\right)=j_{\mu}(x) .
$$

The solution in this case is

$D_{\mu \nu}\left(x-x^{\prime}\right)=-\frac{1}{(2 \pi)^{4}} \int d k \frac{e^{-i k\left(x-x^{\prime}\right)}}{k^{2}}\left[g_{\mu \nu}-\frac{k_{\mu} k_{v}}{k^{2}}\right)$

which satisfies (B5) and (B6) if one makes use of the conservation of the current

$$
k_{v} j^{v}(x)=0 \text {. }
$$

This choice (B7) for $D_{\mu \nu}\left(x-x^{\prime}\right)$ is sometimes called the Landau gauge. Another possible choice is

$$
D_{\mu \nu}\left(x-x^{\prime}\right)=-\frac{1}{(2 \pi)^{4}} \int d k \frac{e^{-i k\left(x-x^{\prime}\right)}}{k^{2}} g_{\mu \nu} .
$$

One can again check that this choice also satisfies (B5) and (B6). Note that whereas in the first case the conservation of the current is needed to satisfy (B6) while (B5) is automatically fulfilled, exactly the opposite is true in the second case.

\section{The Coulomb gauge}

The condition on the potential $\partial^{k} A_{k}^{S}(x)=0$ implies

$$
\int d x^{\prime} \partial^{k} D_{k \mu}\left(x-x^{\prime}\right) j^{\mu}\left(x^{\prime}\right)=0
$$

and transforms (B1) into

$$
\begin{gathered}
\int d x^{\prime} \partial^{k} \partial_{k} D^{0 \mu}\left(x-x^{\prime}\right) j_{\mu}\left(x^{\prime}\right)=j^{0}(x) \\
\int d x^{\prime}\left[\left(\partial^{t} \partial_{t}+\partial^{i} \partial_{i}\right) D^{k \mu}\left(x-x^{\prime}\right)\right. \\
\left.-\partial^{k} \partial_{t} D^{0 \mu}\left(x-x^{\prime}\right)\right] j_{\mu}\left(x^{\prime}\right)=j^{k}(x) .
\end{gathered}
$$

Again, it is easy to check that the following choice satisfies (B10) and (B11):

$$
\begin{aligned}
& D_{k l}\left(x-x^{\prime}\right)=\frac{-1}{(2 \pi)^{4}} \int d k \frac{e^{-i k\left(x-x^{\prime}\right)}}{k^{2}}\left(\delta_{k l}-\frac{k_{k} k_{l}}{\mathbf{k}^{2}}\right), \\
& D_{0 k}\left(x-x^{\prime}\right)=D_{k 0}\left(x-x^{\prime}\right)=0, \\
& D_{00}\left(x-x^{\prime}\right)=-\frac{1}{(2 \pi)^{4}} \int d k \frac{e^{-i k\left(x-x^{\prime}\right)}}{\mathbf{k}^{2}},
\end{aligned}
$$

if the continuity equation is used.

\section{The Dzialochinsky gauge}

If one requires that $A^{0 S}=0$ one gets

$$
\int d x^{\prime} D_{0 \mu}\left(x-x^{\prime}\right) j^{\mu}\left(x^{\prime}\right)=0,
$$

and (B1) becomes

$$
\begin{gathered}
\int d x^{\prime} \partial^{k} \partial_{t} D_{k \mu}\left(x-x^{\prime}\right) j^{\mu}\left(x^{\prime}\right)=j_{0}(x), \\
\int d x^{\prime}\left[\left(\partial^{t} \partial_{t}+\partial^{i} \partial_{i}\right) D_{k \mu}\left(x-x^{\prime}\right) j^{\mu}\left(x^{\prime}\right)\right. \\
\left.-\partial^{l} \partial_{k} D_{l \mu}\left(x-x^{\prime}\right) j^{\mu}\left(x^{\prime}\right)\right]=j_{k}(x) .
\end{gathered}
$$

The choice

$$
\begin{aligned}
& D_{k l}\left(x-x^{\prime}\right)=\frac{-1}{(2 \pi)^{4}} \int d k \frac{e^{-i k\left(x-x^{\prime}\right)}}{k^{2}}\left(\delta_{k l}-\frac{k_{k} k_{l}}{k_{0}^{2}}\right), \\
& D_{k 0}\left(x-x^{\prime}\right)=D_{0 k}\left(x-x^{\prime}\right)=0, \\
& D_{00}\left(x-x^{\prime}\right)=0
\end{aligned}
$$

satisfies (B13) and (B14) using the equation of continuity. Furthermore, one can show explicitly in the self-energy expressions the equivalence of the different gauges, e.g., $\int d x d x^{\prime} j^{\mu}(x) D_{\mu v}\left(x-x^{\prime}\right) j^{v}\left(x^{\prime}\right)$ in the Coulomb gauge and in the Dzialochinsky gauge leads to the same expression:

$$
\begin{aligned}
\int d k d x d x^{\prime}\left[j_{k}(x)\left[\delta^{k l}-\frac{k^{k} k^{l}}{\mathbf{k}^{2}}\right] j_{l}\left(x^{\prime}\right)+j^{0}(x) \frac{k^{2}}{\mathbf{k}^{2}} j^{0}\left(x^{\prime}\right)\right] & \frac{e^{-i k\left(x-x^{\prime}\right)}}{k^{2}} \\
& =\int d k d x d x^{\prime}\left[j_{k}(x)\left[\delta^{k l}-\frac{k^{k} k^{l}}{k_{0}^{2}}\right] j_{l}\left(x^{\prime}\right)\right] \frac{e^{-i k\left(x-x^{\prime}\right)}}{k^{2}}
\end{aligned}
$$




\section{APPENDIX C}

The self-energy corrections are included in the action term $W^{(2)}$ in Eq. (14) containing the factor $\delta\left(E_{n}-E_{m}+E_{r}-E_{s}\right) . A$ priori there are many ways of satisfying this $\delta$ function other than just by Eqs. (15). The energies $E_{n}, E_{m}, E_{r}, E_{s}$ may be discrete or continuous, all four of them, in principle, different, etc. But we wish to attribute these corrections to individual states, hence they must be written in the form $\phi_{n}^{*}(\cdots) \phi_{n}$. This restricts the solutions to those given by Eq. (15) where now the $E$ 's can be discrete or continuous. Any other solution would be of measure zero (for example, three discrete, one continuous energies) or would actually not occur.

\section{APPENDIX D}

The angular integrations in momentum space have been performed according to the formula

$$
\begin{aligned}
\sum_{k, l=1}^{3} \int d \Omega_{k}\left[\delta^{k l}-\frac{k^{k} k^{l}}{\mathbf{k}^{2}}\right] A^{j}\left(\mathbf{k}^{2}\right) B^{l}\left(\mathbf{k}^{2}\right) & \\
& =4 \pi \mathbf{A} \cdot \mathbf{B}-\frac{4 \pi}{3} \mathbf{A} \cdot \mathbf{B} \\
& =\frac{8 \pi}{3} \mathbf{A} \cdot \mathbf{B}
\end{aligned}
$$

which can be proved by explicit integrations.
*Permanent address: Physics Department, University of Colorado, Boulder, CO 80309.

${ }^{\dagger}$ Also at the Interuniversitair Instituut voor Kernwetenschappen.

${ }^{1}$ A. O. Barut, in Foundations of Radiation Theory and Quantum Electrodynamics (Plenum, New York, 1980), p. 165.

${ }^{2}$ A. O. Barut and J. Kraus, Found. Phys. 13, 189 (1983).

${ }^{3} \mathrm{H}$. Bethe and E. Salpeter, Quantum Mechanics of One- and Two-Electron Systems (Plenum, New York, 1977). Compare formula (19.7). The authors make the dipole approximation.
For a discussion of that approximation see Sec. (19. $\gamma$ ), and C.

K. Au and G. Feinberg, Phys. Rev. D 9, 1794 (1974).

${ }^{4}$ A. O. Barut, in Quantum Electrodynamics and Quantum Optics (Plenum, New York, 1984), p. 15.

${ }^{5}$ See, for example, A. O. Barut, Electrodynamics and Classical Theory of Fields and Particles (Dover, New York, 1980).

${ }^{6}$ E. Wichmann and N. Kroll, Phys. Rev. 101, 843 (1956).

${ }^{7}$ E. A. Uehling, Phys. Rev. 48, 55 (1935).

${ }^{8}$ H. Bethe and E. Salpeter, Ref. 3, Sec. 67.

${ }^{9}$ H. Bethe and E. Salpeter, Ref. 3, Sec. 59. 